\title{
On the High Temperature Operation of High Voltage Power Devices
}

\author{
Vasile V.N. Obreja \\ National R\&D Institute for Microtechnology \\ (IMT), P.O. Box 38-160, Bucharest, Romania \\ Tel: +401-490-8212, Fax: +401-490-8238, \\ E-mail:vasileo@imt.ro,vnobreja@hotmail.com
}

\author{
Keith I. Nuttall \\ Department of Electrical Engineering and \\ Electronics, The University of Liverpool, \\ L693BX, Liverpool, UK, Tel: 0151 -794-4508 \\ E-mail:ee16@liverpool.ac.uk
}

\begin{abstract}
The surface component of reverse current is a serious limitation for the operation of high voltage/current devices at high junction temperature. This component may induce reverse current-voltage instability of a PN junction followed by device failure during high temperature operation. Experimental results are presented for silicon rectifier diodes revealing the influence of the junction surface leakage current on the reverse I-V characteristics. Known results for silicon carbide PIN diodes are considered for comparison. Available PN junction passivation methods at this time are not effective enough in controlling the junction surface leakage current.

Improved surface passivation techniques are required to achieve reliable device operation, especially above $200^{\circ} \mathrm{C}$.
\end{abstract}

\section{Introduction}

Silicon high voltage bipolar or MOS devices cannot operate reliably at junction temperature higher than 175 $-200{ }^{\circ} \mathrm{C}$. Although high temperature performance is expected from $\mathrm{SiC}$, commercial devices with a permissible junction operation temperature above $200{ }^{\circ} \mathrm{C}$ are still not available. The first Schottky barrier diodes with a rated current from $1 \mathrm{~A}$ to $10 \mathrm{~A}$ and blocking voltage rated from $300 \mathrm{~V}$ to $600 \mathrm{~V}$ which appeared on the market in 2001 have a maximum junction operation temperature limited to $175{ }^{\circ} \mathrm{C}$. SiC based PIN diodes have been demonstrated with a reverse voltage up to 6.2 $\mathrm{kV},[1-2]$, but are still not available as commercial devices. As reported in [2] surface phenomena from the junction peripheral surface may govern the diode behavior at reverse bias voltage and low forward voltage.

Even though silicon device technology has a very mature status, the reverse I-V characteristics of silicon power PN junctions may still be controlled by surface effects, [3-4]. PN junctions are vital building cells of modern bipolar or MOS semiconductor devices. Power devices whose PN junction passivation is based on Si$\mathrm{SiO}_{2}$ interface manifest reverse current-voltage instability which may be followed by failure at elevated temperature sometimes even before $200{ }^{\circ} \mathrm{C}$, [5]. Analysis of defective devices after operation at high junction temperature, $T_{j}$, has revealed electrical short-circuits accompanied by crystalline lattice damage at the junction peripheral surface.

The purpose of this work is to show that electrical phenomena at the junction peripheral surface are still a significant factor that is preventing the full potential of silicon devices operated at junction temperatures above $200{ }^{\circ} \mathrm{C}$ from being realized.

\section{Experimental results. Discussion}

Typical results that reveal the influence of junction surface effects on the reverse I-V characteristics are shown in Fig.1. This data relates to a mesa type silicon $\mathrm{P}^{+} \mathrm{NN}^{+}$structure of about $16 \mathrm{~mm}^{2}$ area and passivated with organic dielectric (silicone rubber). This passivation method has the advantage that for the same structure, the passivation dielectric layer can be easily removed and the passivation process repeated.

Planar oxide and glass passivated junctions of about the same area and breakdown reverse voltage as those considered in Fig.1 have also been investigated. For some of them the passivating oxide was removed and a re-passivation by using organic dielectric was performed. It has been found that the state of the silicon junction surface in direct contact with the dielectric layer is of great significance for $\mathrm{I}-\mathrm{V}$ reverse characteristic stability at $\mathrm{T}_{\mathrm{j}}$ higher than $200{ }^{\circ} \mathrm{C}$ whilst the nature of the dielectric passivation material (organic or inorganic) is of less importance. The results in Fig.1 also show a significant reduction in the value of reverse current, $I_{R}$, takes place after heat treatment at $300{ }^{\circ} \mathrm{C}$ and that since the same behavior is repeated with the second passivation and anneal cycle, then this must be attributed to a reduction in surface related current. Fig. 1 also shows that a low level of $I_{R}$ near room temperature does not necessarily provide a low $I_{R}$ at high $T_{j}$. Further experiments taking into account a correlation of the $I_{R}$ level with the junction area and its perimeter have indicated that the I-V characteristics in Fig.1 after heat treatment at $300{ }^{\circ} \mathrm{C}$ are still influenced by the surface component.

Investigated glass or oxide passivated junctions of available commercial devices with a silicon die of about 

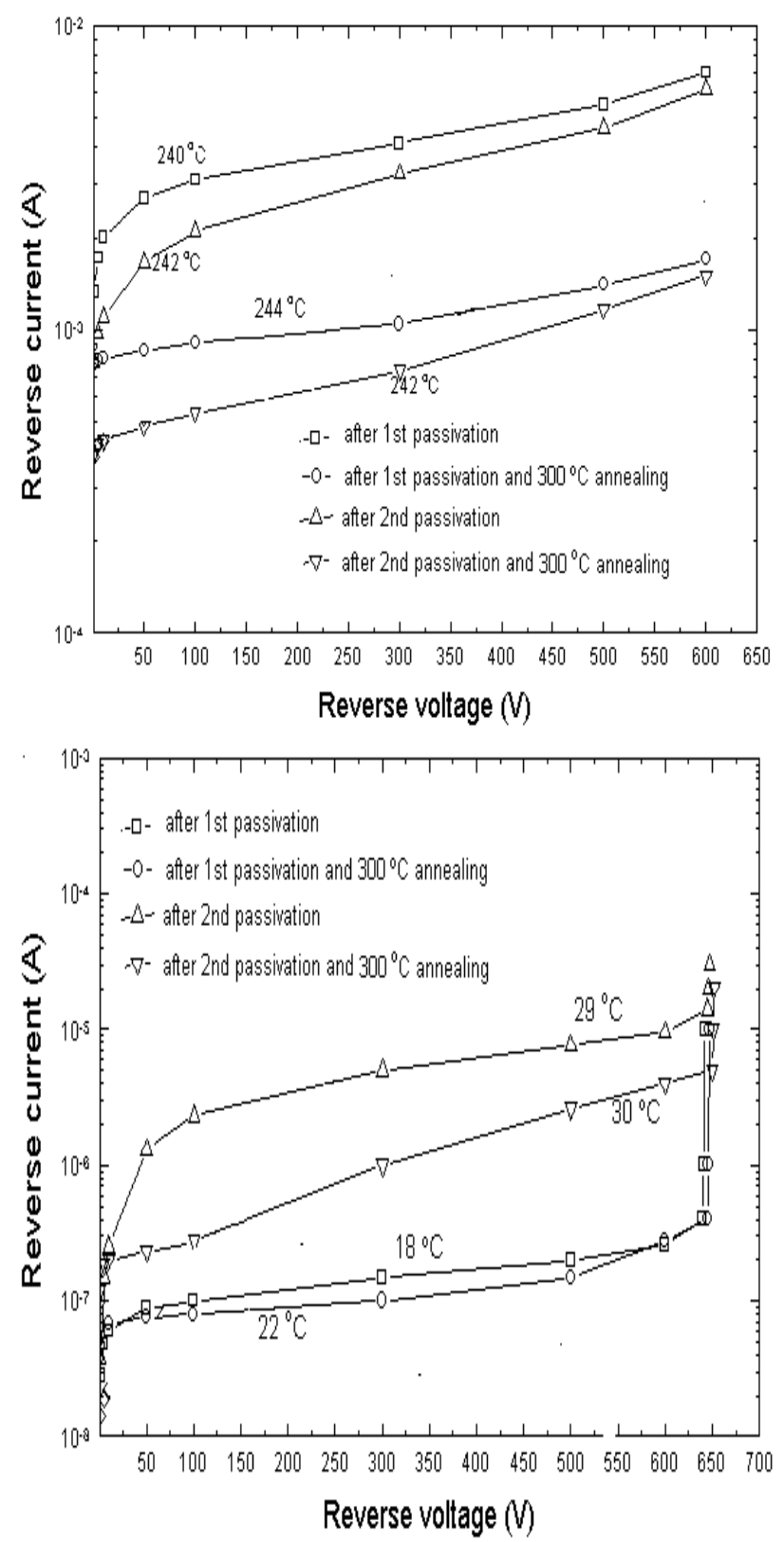

Figure 1. Current-voltage characteristics for a silicon controlled-avalanche diode structure with organic dielectric passivation near room temperature and above $200^{\circ} \mathrm{C}$ junction temperature

the same dimensions, breakdown voltage and charge carriers lifetime as that used in Fig. 1, exhibited $I_{R}$ values above $200{ }^{\circ} \mathrm{C}$ not less than the lowest level shown in this figure. Consequently, the $I_{R}$ level of junctions passivated with inorganic dielectric is not necessarily less than those passivated with organic dielectric and may be also controlled by surface phenomena.

Other examples that demonstrate the influence of the surface component on the level of $I_{R}$ are shown in Fig.2. This time a mesa $\mathrm{P}^{+} \mathrm{NN}^{+}$rectifier silicon structure of 16 $\mathrm{mm}^{2}$ area but with an expected bulk breakdown voltage higher than $1500 \mathrm{~V}$ has been used and the graphs have been drawn with logarithmic voltage scales. The different I-V reverse characteristics shown correspond to three different re-passivations of the same silicon structure. The characteristics plotted after the 1st passivation relate to the situation when some contamination from the surrounding manufacturing ambience was permitted after the cleaning of the junction surface. Reverse characteristics for the 2nd and the 3rd re-passivations relate to a passivation process that was completed without allowing any influence from the surrounding manufacturing ambience. Nonetheless, some acid traces remained after the junction surface cleaning during the passivation process, more for the 2 nd and less for the the 3rd re-passivation.

Fig. 2 shows that the surface component of $I_{R}$ has a significant influence not only on its level at room temperature and above, but also on the obtained breakdown voltage value. This time, significant voltage dependence of $I_{R}$ (soft surface breakdown characteristic) is evident at room temperature well below the expected value of the bulk breakdown. At higher junction temperature the curvature of the characteristic towards $1000 \mathrm{~V}$ is significantly attenuated. As in the case of Fig.1 a reduced voltage dependence of $I_{R}$ at room temperature observed in Fig.2 (which may be favorable in reaching the bulk breakdown value determined at low current), does not provide low $I_{R}$ values at high $T_{j}$. In practice it is known that for higher reverse working voltages, above $1000 \mathrm{~V}$, it is more difficult to reach the bulk breakdown voltage. Silicon controlled-avalanche diodes of breakdown voltage above $2000-3000 \mathrm{~V}$ are not easily realized.

While the level of reverse current at high junction temperature for the 1st passivation in Fig.2 is comparable with the corresponding one for similar commercial silicon diodes available on the market, for the other two passivation situations the level of leakage current is significant lower. It has also been found that the blocking I-V characteristic of the drain junction of some investigated high voltage commercial power MOS devices of about the same area as the diode structure used in Fig.2, do not exhibit lower $I_{R}$ values at high $T_{j}$ than it is shown in this figure. A saturation tendency of $I_{R}$ at lower $V_{R}$ and high $T_{j}$ observed in Fig.2 for the $1^{\text {st }}$ and $3^{\text {rd }}$ passivations cannot be considered evidence for the bulk diffusion component dominance because even lower $I_{R}$ values are obtained for the $2^{\text {nd }}$ passivation case where visible voltage dependence is manifested. Consequently, in spite of significantly reduced $I_{R}$ values at high $T_{j}$ obtained in Fig.2 its level continues to be under the influence of the surface component. Nonetheless, excessive high surface leakage current is prevented for the $2^{\text {nd }}$ and $3^{\text {rd }}$ passivation case.

High temperature reverse bias (HTRB) tests at $250{ }^{\circ} \mathrm{C}$ and $300 \mathrm{~V}$ for 250 hours have indicated good stability of the devices passivated in the same conditions as for the 2nd or the 3rd re-passivation cycle but no device from the first passivation cycle survived. These devices exhibited a relatively high initial $\mathrm{I}_{\mathrm{R}}$ level at $250{ }^{\circ} \mathrm{C}$ and 

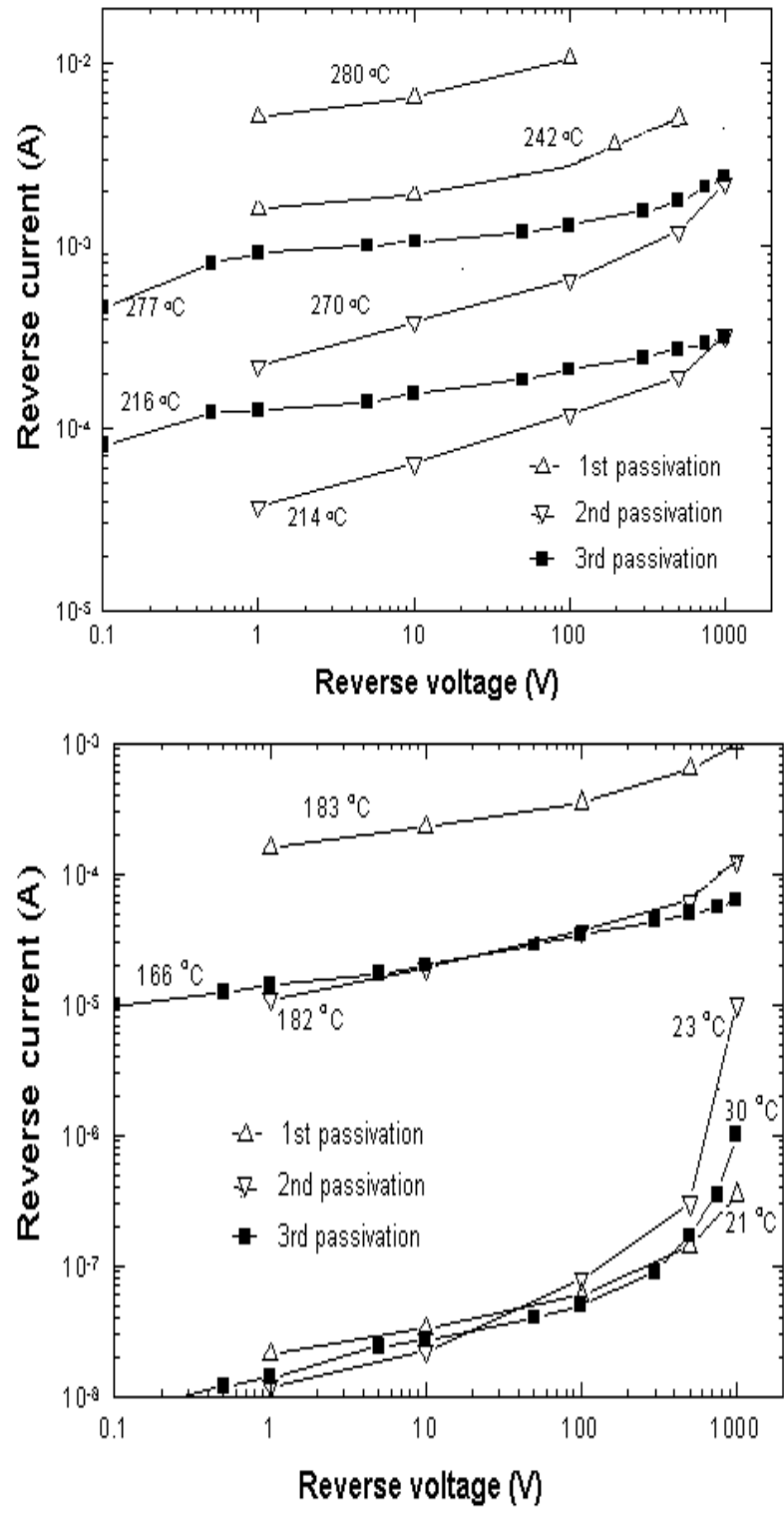

Figure 2. Electrical characteristics of a silicon high voltage rectifier diode passivated with organic dielectric; $\mathbf{1}^{\text {st }}$ passivation: permitted exposure of the junction peripheral surface to the surrounding manufacturing ambience; $2^{\text {nd }}$ and $3^{\text {rd }}$ passivations: without exposure to the ambient but with acid traces, more for the second and less for the third.

$300 \mathrm{~V}$ of $6-8 \mathrm{~mA}$. After the test, electrical shortcircuits were found, caused by local current conducting at the junction peripheral surface. Devices from the $2^{\text {nd }}$ and $3^{\text {rd }}$ passivation cycle that were subjected to HTRB tests had a reverse current level of $0.5-0.7 \mathrm{~mA}$ at 250 ${ }^{\circ} \mathrm{C}$ and $300 \mathrm{~V}$ prior to the test. After the tests no significant change in the level of $\mathrm{I}_{\mathrm{R}}$ was found. It is likely that local overheating takes place at the junction peripheral surface due to excessive local power dissipation, particularly if it is considered that the surface current is unlikely to be uniformly distributed around the junction perimeter.
As a consequence, reliable operation of silicon high voltage diodes at junction temperature above $200{ }^{\circ} \mathrm{C}$ is only possible when the surface component of the reverse current is kept at a low level by a suitable junction passivation process. Suitably chosen organic dielectrics for junction passivation may be more efficient at high temperatures than the presently used inorganic dielectrics.

The experimental results presented in this work were taken on high carrier lifetime PN junctions i.e. the density of bulk generation -recombination centers is low. However, for silicon fast recovery PN junctions the bulk component of $I_{R}$ may become the primary component at $\mathrm{T}_{\mathrm{j}}$ higher than $125-150{ }^{\circ} \mathrm{C}$, [4]. Nevertheless even in this case, the surface component can still be a cause for reverse I-V characteristic degradation at elevated temperature.

To our knowledge PIN diodes based on $\mathrm{SiC}$ and with an area larger than $10 \mathrm{~mm}^{2}$ are still not available even as demonstration devices. However, a significantly lower level of $I_{R}$ for $\mathrm{SiC}$ based devices is expected because the carrier intrinsic concentration for this material is orders of magnitude lower than for silicon. Nevertheless it has already been observed in the literature that the experimental values for $I_{R}$ are orders of magnitude higher than predicted. Near room temperature, I-V reverse electrical characteristics of similar shape and visible voltage dependence of $I_{R}$ as shown in Fig.2 have been reported. At high $T_{j}$ the same saturation tendency is observed. High excessive $I_{R}$ is possible for a SiC PIN diode in comparison with another diode from the same wafer and with the same physical parameters. Published experimental reverse voltage characteristics for $4 \mathrm{H}-\mathrm{SiC}$ or $6 \mathrm{H}-\mathrm{SiC}$ diodes are often limited to less than $250{ }^{\circ} \mathrm{C}$ and voltages significantly lower than those given for the room temperature, $[1,2]$. Furthermore, the practice of expressing PN junction reverse leakage as a current density $\left(\mathrm{A} / \mathrm{cm}^{2}\right)$ can be misleading in the presence of a significant contribution from surface current.

Evidently, high temperature surface passivation is an issue that will need to be addressed to successfully realize the full potential not only of silicon, but of $\mathrm{SiC}$ as well.

\section{PN junction physical model}

The experimental results presented in Figs.1-2 can be understood by taking into account surface electrical phenomena of the types shown in Fig.3. Either a surface inversion, depletion or accumulation layer may cause surface leakage current at reverse bias voltage. The surface current component is dependent on the carrier intrinsic concentration. Consequently, for silicon based junctions a significant higher level of $I_{R}$ is expected than for semiconductors with higher band gap energy.

Besides the bulk component of $I_{R}$ uniformly distributed over the junction area, the surface component may be non-uniformly distributed over the junction perimeter so that reverse current crowding may be favored in some 


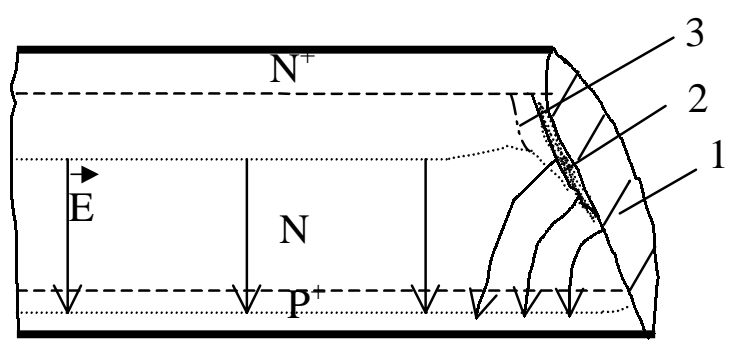

a)

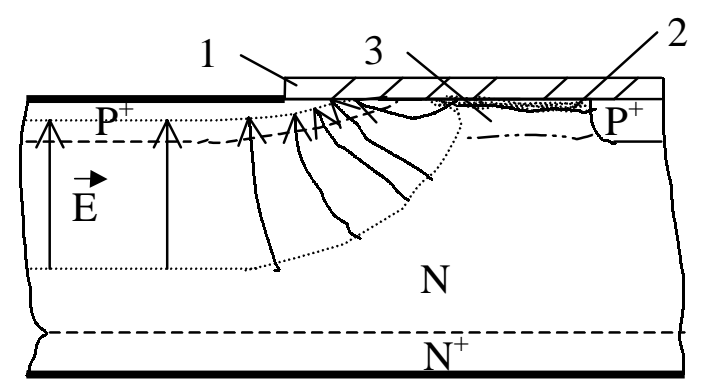

b)

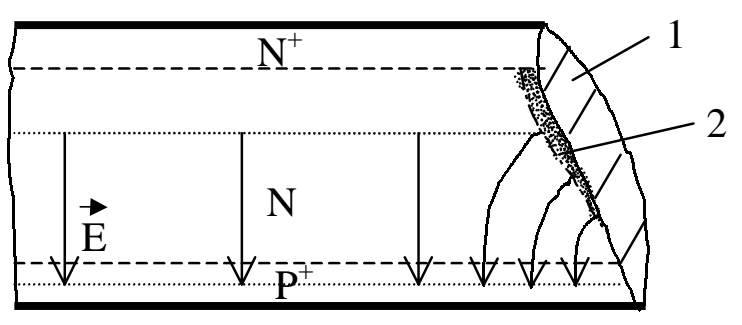

c)

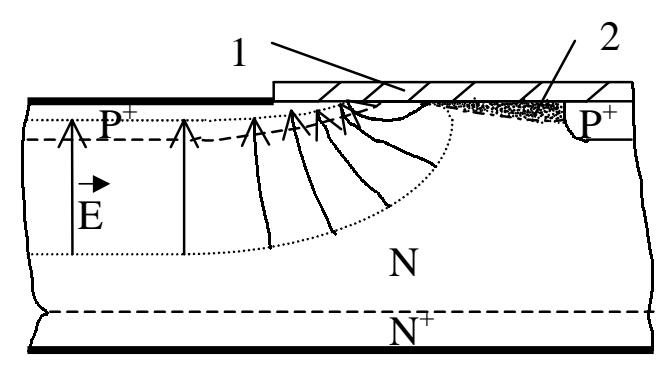

d)

Figure 3. Surface space charge layers in silicon PN junctions under reverse bias voltage; inversion and depletion layer in mesa (a) and planar (b) PN junction; accumulation layer in mesa (c) and planar (d) PN junction;

1- passivation dielectric layer; 2- inversion or accumulation layer; 3 - depletion layer; local regions. Ideally, the junction passivation process would prevent a surface space charge layer forming to assure device ability for operation at $T_{j}$ higher than 200 ${ }^{\circ} \mathrm{C}$. In practice a surface depletion or inversion layer may be responsible for excessive high $I_{R}$ at high $T_{j}-$ such as in the case in Fig.1 before annealing or in Fig.2 $\left(1^{\text {st }}\right.$ passivation $)$ whilst a charge accumulation layer can reduce the surface leakage current. This may account for the behaviour observed in Fig. $1 \& 2$. With a decrease in the doping concentration of the starting silicon material necessary to obtain high working voltages, the formation of a surface space charge layer at the semiconductor surface is more difficult to control. Control difficulties may be also encountered for $\mathrm{SiC}$ due to a significant lower intrinsic concentration. More influence on the level of $I_{R}$ at high $T_{j}$ or on the obtained breakdown voltage value is expected. A weak accumulation layer obtained by suitable junction passivation could be acceptable for high junction temperature operation providing low level of $I_{R}$ from low to high reverse voltage. The annealing process and silicon surface treatment with acid traces whilst avoiding contamination from the surrounding ambient are thought to be instrumental in creating these conditions.

\section{Conclusion}

The results presented indicate that high temperature operation of silicon power devices may be still affected by a significant surface leakage current component. Presently available junction passivation based on the Si$\mathrm{SiO}_{2}$ interface does not appear to provide an adequate control of leakage current needed to fully realize the potential of silicon at temperatures above $200{ }^{\circ} \mathrm{C}$. This is an issue that will be particularly relevant to the successful introduction of $\mathrm{SiC}$ devices.

\section{References}

[1] K. Fujihira, S. Tamura, T. Kimoto, and H..Matsunami , "Low-Loss, High-Voltage 6H-SiC Epitaxial p-i-n Diode", IEEE Transactions on Electron Devices,vol.49, No.1, 2002, pp. $150-154$

[2] T. Kimoto, N. Miyamoto, and H. Matsunami, "Performance Limiting Surface Defects in $\mathrm{SiC}$ Epitaxial p-n Junction Diodes", IEEE Transactions on Electron Devices, vol.46, No.3,1999, pp.471 - 477

[3] V. Obreja, "On the Leakage Current of Present-Day Manufactured Semiconductor Junctions", Solid-State Electronics, vol.44, No.1, .2000, p. 49-57

[4] V. Obreja , "An experimental investigation on the nature of reverse current of power silicon PN junctions" IEEE Transactions on Electron Devices, vol.49, No.1, 2002, pp.155 163

[5] J.R. Trost, R.S. Ridley, M. Kamal Khan and T.Grebs, “The effect of charge in junction termination extention passivation dielectrics", Proc.11 $1^{\text {th }}$ Int. Symp. Power Semiconductor Devices and ICs (ISPSD), 1999, pp.189-192 